\title{
RESISTANCE TRAINING INDUCES ANTI-ATHEROGENIC EFFECTS ON METABOLOMIC PATHWAYS
}

\section{Heikki V. Sarin ${ }^{1,2 *}$, Juha P. Ahtiainen ${ }^{3 *}$, Juha J. Hulmi ${ }^{3,4}$, Johanna K. Ihalainen ${ }^{3,5}$, Simon Walker $^{3}$, Maria Küüsmaa-Schildt ${ }^{3}$, Markus Perola ${ }^{1,2}$, Heikki Peltonen ${ }^{3}$}

${ }^{1}$ Genomics and Biomarkers Unit, Department of Health, National Institute for Health and Welfare, Helsinki, Finland

${ }^{2}$ Research Program for Clinical and Molecular Metabolism, Faculty of Medicine, University of Helsinki

${ }^{3}$ NeuroMuscular Research Center, Faculty of Sport and Health Sciences, University of Jyväskylä, Jyväskylä, Finland

${ }^{4}$ Department of Physiology, Faculty of Medicine, University of Helsinki

${ }^{5}$ Swedish Winter Sports Research Centre, Department of Health Sciences, Mid Sweden University, Östersund, Sweden

*These authors contributed equally to this work

Corresponding author: Heikki V. Sarin, M.Sc., B.Med.

Genomics and Biomarkers Unit, The Department of Public health solutions

National Institute for Health and Welfare (THL)

P.O. Box 30 (Mannerheimintie 166)

FIN - 00271 Helsinki

Finland

Email: heikki.sarin@thl.fi

Telephone: (358) 44-0764808 
$\mathbf{a}$

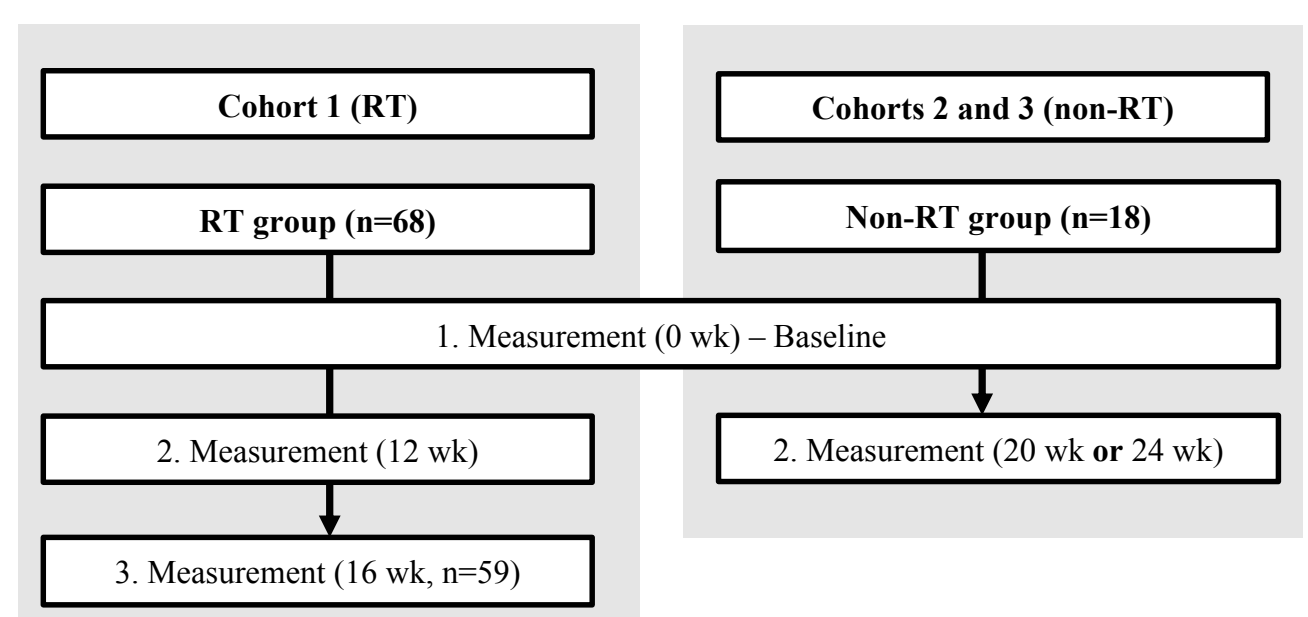

b
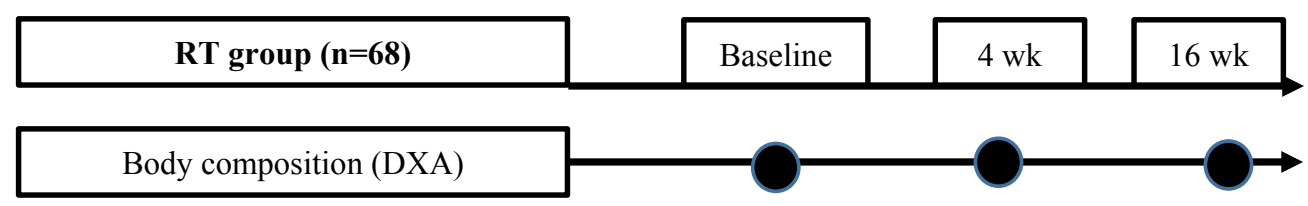

Muscle strength (leg press 1RM)

Venous blood samples

Dietary intake (4 day food diary)

c

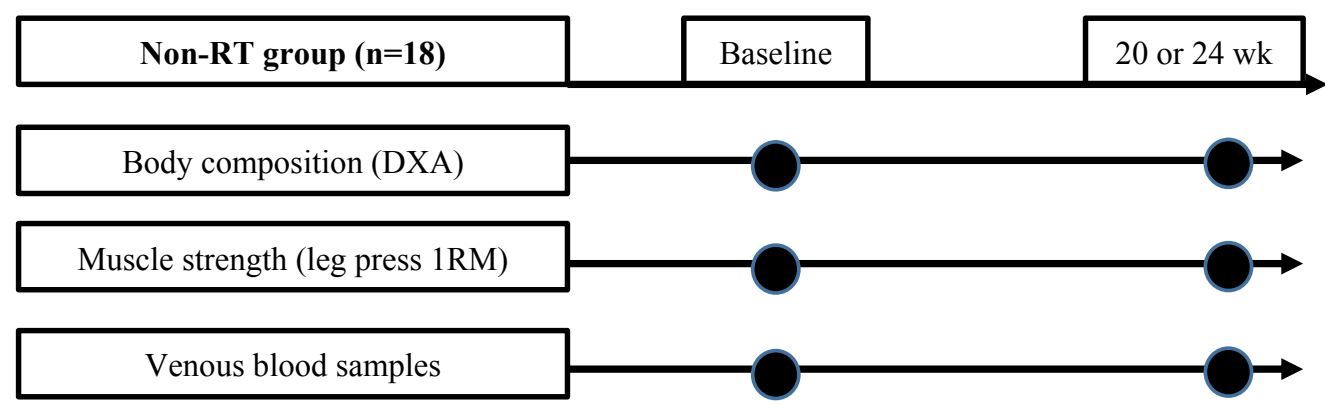

Supplementary Figure 1. Flowchart of study design. The duration of the fully supervised resistance training intervention for the RT group was 16 weeks. Measurements were performed at baseline (PRE), after 4 weeks (POST-4wk), and after 16 weeks (POST-16wk) of resistance training. The non-RT group was measured at baseline (PRE) and after (POST-control) 20weeks $(\mathrm{n}=8)$ or 24 -weeks $(\mathrm{n}=10)$ into the study periods. The non-RT group was formed using data from two previously collected cohorts which is why the follow-up times vary between groups. Panel A depicts overall study design whereas panels B (RT group) and C (non-RT group) depict at which time points each measurements were performed. 